\title{
HemoCue against Coulter LH-750 in the estimation of hemoglobin levels of blood donors in mobile collection settings: a comparative study
}

\author{
Sultan A.M. Saghir, ${ }^{1,5}$ Amer A. Almaiman, ${ }^{2,3}$ Aishah K.A. Shatar, ${ }^{2}$ Norris Naim, ${ }^{4}$ Huda S. Baqir ${ }^{2}$ \\ ${ }^{1}$ Department of Medical Analysis, Princess Aisha Bint Al-Hussein, College of Nursing and Medical Sciences, Al-Hussein Bin Talal \\ University, Ma`an, Jordan. \\ ${ }^{2}$ Advanced Medical and Dental Institute, Universiti Sains Malaysia, Bertam, Malaysia \\ ${ }^{3}$ Unaizah College of Pharmacy, Qassim University, Qassim, Saudi Arabia \\ ${ }^{4}$ Pusat Darah Negara, Kuala Lumpur, Malaysia \\ ${ }^{5}$ Department of Pharmacology, School of Pharmaceutical Sciences, Universiti Sains Malaysia, Penang, Malaysia
}

\section{ABSTRACT}

Background: The fast and outpatient setting for a determination of the hemoglobin ( $\mathrm{Hb}$ ) level is a well-recognized prerequisite to detect anemia in blood donors. This study aimed to evaluate the performance of the HemoCue methods (HemoCue B-Hb and HemoCue-301) against Coulter LH-750 as a reference method for Hb determination.

Methods: This study was an experimental cross-sectional study. It includes 455 blood samples that were collected from volunteer blood donors between January 15, 2010 and February 15, 2011. The performance of the three methods and their comparisons were assessed using the analysis of coefficients of variation (CV), linear regression, and mean difference. Correlation coefficient and Bland-Altman plots were drawn to compare the two HemoCue measurements and the automated cell analyzer against each other and to evaluate their results. The Hb concentrations were compared using the concordance correlation coefficient.

Results: The findings exhibited that the CV for the three methods Coulter LH-750, HemoCue B-Hb, and HemoCue-301 were $0.60 \%$, $0.72 \%$, and $0.92 \%$, respectively. A statistically significant difference was observed between the means of the Hb measurements for the three methods ( $\mathrm{p}<0.001)$. The HemoCue B-Hb and HemoCue-301 methods showed the best agreement, and the Coulter LH-750 method gave a lower Hb value compared with the two HemoCue methods. The results showed a positive correlation of HemoCue $\mathrm{Hb}$ results compared with the reference method.

Conclusion: All three methods provide a good agreement for $\mathrm{Hb}$ determination. The new device HemoCue-301 was found to be more accurate compared with HemoCue B-Hb and Coulter LH-750.

Keywords: blood donation, Coulter LH-750, HemoCue, hemoglobin

pISSN: 0853-1773 • eISSN: 2252-8083 • https://doi.org/10.13181/mji.v27i4.2635 • Med J Indones. 2018;27:250-5

- Received 07 Feb 2018 • Accepted 12 Sep 2018

Corresponding author: Sultan A.M. Saghir

sultan_a1976@yahoo.com

Copyright @ 2018 Authors. This is an open access article distributed under the terms of the Creative Commons Attribution-NonCommercial 4.0 International License (http://creativecommons.org/licenses/by-nc/4.0/), which permits unrestricted non-commercial use, distribution, and reproduction in any medium, provided the original author and source are properly cited. 
The determination of the hemoglobin (Hb) level of prospective blood donors is a wellrecognized prerequisite in most countries, and in fact, it is the only laboratory screening test routinely performed before blood donation. The reasons for performing such a test are to protect donors with low $\mathrm{Hb}$ levels from being critically anemized and to make sure that the collected blood units meet the essential standards for $\mathrm{Hb}$ content. ${ }^{1}$ It is, therefore, very important to have a reliable method for $\mathrm{Hb}$ determination to ensure that only non-anemic individuals are accepted as donors. ${ }^{2}$ The Hb cut-off value for blood donation is $12.5 \mathrm{~g} / \mathrm{dl}^{3}$

The World Health Organization and the International Nutritional Anemia Consultative Group have established the reference values of $\mathrm{Hb}$ concentration to define anemia with considerations to age, sex, and certain physiologic circumstances such as pregnancy. ${ }^{4}$ Donor eligibility criteria are designed to protect both the donor and the recipient. ${ }^{5}$ Anemia can be diagnosed by measuring the $\mathrm{Hb}$ concentration in venous or capillary blood, and such measurement is used at the population level to estimate the prevalence of anemia, allocate resources, and target intervention programs to vulnerable groups. At the individual level, it is used to screen for participation in programs and evaluate response to interventions. ${ }^{6,7}$ There are various methods for $\mathrm{Hb}$ estimation, and they vary from a simple paper scale to measurements by a spectrophotometer; each one has its own advantages and limitations. ${ }^{2}$

At the mobile collection centers, where a large number of people need to be screened in a short time, a quick, inexpensive, and easy method is needed. One of such methods is the copper sulfate method, which is based upon the observation that the specific gravity of blood is greatly influenced by its erythrocytes volume. The specific gravity dependent-method is the traditional method used for donor screening at most of blood collection centers. ${ }^{8}$ The HemoCue system employs the principle of converting $\mathrm{Hb}$ to azide methemoglobin, which is measured at 565 and $880 \mathrm{~nm}$ to ensure automatic compensation for turbidity (due to lipemia or leukocytosis). ${ }^{9,10}$ The new HemoCue equipment (HemoCue-301) is now available at Pusat Darah Negara (PDN). The manufacturer claims that it is more accurate than the older HemoCue equipment (HemoCue B-Hb), which is currently being used at mobile collection centers (informal communication from PDN in Kuala Lumpur).

The new HemoCue equipment (HemoCue-301) has also been offered to the PDN. However, an assessment of its accuracy is needed before a decision on any purchasing can be made. Thus, this study was designed to compare the two HemoCue methods and to evaluate their efficacy in $\mathrm{Hb}$ determination compared with the automatic blood analyzer (Coulter LH-750) as the reference method.

\section{METHODS}

An experimental cross-sectional study utilizing 455 blood samples was carried out in a blood donation setting during mobile collection in PDN, Kuala Lumpur, to evaluate the quality of the two methods of $\mathrm{Hb}$ estimation (HemoCue $\mathrm{B}-\mathrm{Hb}$ and HemoCue-301) and to compare with Coulter LH-750 as a reference method.

All blood samples were collected between January 2011 and February 2011 from volunteer blood donors. Consent forms were obtained from the participants. Ethical approval for the study was obtained from the research ethics committee at the Advanced Medical and Dental Institute, Universiti Sains Malaysia.

$\mathrm{Hb}$ concentration was measured within 24 $\mathrm{h}$ after sample collection to avoid any discrepancies in the results due to prolonged storage. Ethylenediaminetetraacetic acid vacutainer tubes were used to collect $2 \mathrm{ml}$ of venous blood samples to be used for the $\mathrm{Hb}$ determination by different methods. Blood samples were collected under identical conditions from donors for Hbassessment: first by HemoCue B-Hb, then by HemoCue-301, and lastly by the automated analyzer (Coulter LH-750) after $8 \mathrm{~h}$. All instruments were run, calibrated, and controlled according to recommendations from the manufacturers.

The chosen donors successfully passed the donor selection guidelines of PDN (2008), Kuala Lumpur, Malaysia. All participants were between 18 and 65 years old for both men and women and have a minimum weight of $45 \mathrm{~kg}$. They appear in good health with no bleeding disorders, recent illness or medication taken, and have slept a minimum of five 
Table 1. Coefficient variation for using HemoCue B-Hb, HemoCue-301, and Coulter LH-750 after ten times $\mathrm{Hb}$ measurement for one sample

\begin{tabular}{lcc}
\hline Method & $\mathrm{Hb}$ g/dl $($ Mean \pm SD) & CV \\
\hline Coulter LH 750 & $13.2 \pm 0.08$ & 0.60 \\
HemoCue B-Hb & $13.9 \pm 0.10$ & 0.72 \\
HemoCue-301 & $14.1 \pm 0.13$ & 0.92 \\
\hline
\end{tabular}

$\mathrm{SD}=$ standard deviation and $\mathrm{CV}=$ coefficient of variation

hours. The interval between their last donations should not be less than 8 weeks for whole blood and not less than 2 weeks for plasma or platelet. All donors who have no consent form or are not fit to the donor criteria of PDN were excluded.

\section{Statistical analysis}

Statistical analysis was performed using Statistical Product and Service Solutions (SPSS) version 16. Correlation coefficient and BlandAltman plots were drawn to compare the two HemoCue measurements and the automated cell analyzer against each other and to evaluate their results. The $\mathrm{Hb}$ concentrations were compared using the concordance correlation coefficient. ${ }^{11,12}$ This coefficient measures the strength of the relation between the two estimates, as well as the deviation from the $45^{\circ}$ line through the origin (line of equity)..$^{13}$ The coefficient is considered a good method for comparing the precision, reliability, and accuracy of test results. ${ }^{14}$

\section{RESULTS}

There were 455 total subjects in this study: $275(60.4 \%)$ are men and 180 (39.6\%) are women. Of the participants, $120(26.3 \%)$ are Malay, 278 (63\%) are Chinese, 22 (4.8\%) are Indian, and $35(7.6 \%)$ are others. The age of the subjects ranges from 18 to 59 years. The age variables were divided into four groups: group 1 (18-29 years old), group 2 (30-39 years old), group 3 (40-49 years old), and group 4 (50-59 years old). The subjects' distribution within each age group is $420,17,12$, and 6 , respectively. The distribution of the $\mathrm{ABO}$ blood group of the subjects was $170(37.4 \%)$ O+ve blood groups, 15 (3.3\%) O-ve blood groups, 109 (24\%) A+ve blood groups, 7 (1.5\%) A-ve blood groups, 113 (24.8\%) $\mathrm{B}+\mathrm{ve}$ blood groups, $9(2 \%) \mathrm{B}$-ve blood groups, $30(6.6 \%) \mathrm{AB}+\mathrm{ve}$ blood groups, and $2(0.4 \%)$
Table 2. Hemoglobin level as determined using three methods $(n=455)$

\begin{tabular}{lcc}
\hline Method & Mean \pm SD & Range \\
\hline Coulter LH-750 & $13.9 \pm 1.6$ & $9.2-17.3$ \\
HemoCue B-Hb & $14.9 \pm 1.4$ & $12.7-17.9$ \\
HemoCue-301 & $14.6 \pm 1.3$ & $10.7-17.8$ \\
\hline
\end{tabular}

$\mathrm{SD}=$ standard deviation

AB-ve blood groups. The three methods used for measuring $\mathrm{Hb}$ were checked for normality using the Kolmogorov-Smirnov formula. All the statistic values obtained for the Coulter LH-750, HemoCue $\mathrm{B}-\mathrm{Hb}$, and HemoCue-301 were significantly less than $0.05(0.001,0.002$, and $<0.001$, respectively); thus, it was concluded that all the $\mathrm{Hb}$ results were normally distributed. During $\mathrm{Hb}$ screening using the Coulter LH-750 and the two HemoCue methods, quite a few were rejected because of the decrease of $\mathrm{Hb}$ readings (less than $12.5 \mathrm{~g} / \mathrm{dl}$ ).

In this study, the $\mathrm{Hb}$ level was measured 10 times using the three methods from a single blood sample in order to evaluate and determine the coefficient of variation (CV) for each method. The CV for the Coulter LH-750, HemoCue-301, and HemoCue B-Hb methods were $60 \%, 72 \%$, and $92 \%$, respectively (Table 1 ). Statistical differences were detected for all these parameters. However, we observed that the Coulter method showed the lowest mean $(13.9 \mathrm{~g} / \mathrm{dl})$ and the lowest range (9.2-17.3 g/dl) compared with both the HemoCue $\mathrm{B}-\mathrm{Hb}$ (mean=14.9 and range=12.7-17.9 $\mathrm{g} / \mathrm{dl}$ ) and HemoCue-301 methods (mean=14.6 and range $=10.7-17.8 \mathrm{~g} / \mathrm{dl}$; Table 2).

The correlation coefficient for pairs of methods from the linear regression analysis of the $\mathrm{Hb}$ determination for the three methods was evaluated. Table 3 shows the parameters for this analysis and indicates that every pair had a good correlation coefficient (range from 0.76 to 0.85 ). This result was expected since all three methods were designed to measure the same parameter (Hb level in $\mathrm{g} / \mathrm{dl}$ ).

For instance, this could indicate whether or not greater variability could be associated with a particular range of $\mathrm{Hb}$ determination and thus suggest a lack of precision associated with that $\mathrm{Hb}$ range. Therefore, we decided to 
Table 3. Linear regression analysis between every pair of the three methods used for $\mathrm{Hb}$ determination $(\mathrm{n}=455)$

\begin{tabular}{lcc}
\hline Reference method & Testing method & $\begin{array}{c}\text { Pearson } \\
\text { correlation }\end{array}$ \\
\hline Coulter LH-750 & HemoCue B-Hb & 0.84 \\
Coulter LH-750 & HemoCue-301 & 0.76 \\
HemoCue B-Hb & HemoCue-301 & 0.85 \\
\hline
\end{tabular}

evaluate the agreement of the three methods of $\mathrm{Hb}$ determination using the approach proposed by Bland and Altman. This approach assumes that if two methods are to agree, then the mean of the difference between every paired determination will not be statistically different from zero. The Bland and Altman approach permitted us to estimate the limit of agreement between any two methods. These limits are shown in Table 4. The pair Coulter/HemoCue B-Hb, Coulter/HemoCue-301, and HemoCue $\mathrm{B}-\mathrm{Hb} / \mathrm{HemoCue}-301$ gave a limit of agreement of $1.72,2.1$, and $1.5 \mathrm{~g} / \mathrm{dl}$, respectively. Therefore, these are the methods that agree on $\mathrm{Hb}$ measurements. Pairs of methods that involved the HemoCue methods gave a mean of the difference statistically different from zero $(\mathrm{p}<0.001)$.

The pair Coulter/HemoCue B-Hb has a mean of the difference of 1.0, while pairs involving Coulter/HemoCue-301 and HemoCue B-Hb/ HemoCue-301 have 0.7 and 0.3 , respectively (Table 4). The limit of agreement reflects the dispersion of the data around the means of the differences (Figures 1-3). Individual comparisons were made between every two methods using paired t-test from the three methods used for measuring $\mathrm{Hb}$. Statistically significant differences were detected when we compared Coulter LH-750 with either HemoCue methods or when we compared both HemoCue methods against each other $(\mathrm{p}<0.001)$. Figure 1 shows that the $\mathrm{Hb}$ values are closed to the mean and displays that there is a strong correlation between the $\mathrm{Hb}$ results for both methods. In addition, most of the $\mathrm{Hb}$ values are found between 12.0 and $17.8 \mathrm{~g} / \mathrm{dl}$. A few results are scattered away from these two values, and there are no values below $12.0 \mathrm{~g} / \mathrm{dl}$ except for two values only. The dispersion of data in Figure 2 demonstrates that the $\mathrm{Hb}$ values are restricted between 11.5 and 16.3 $\mathrm{g} / \mathrm{dl}$ and few results are exceeding this range. But most of the results are between 14.0 and $16.0 \mathrm{~g} / \mathrm{dl}$ (Figure 2). Also, this figure exhibits that there is a strong positive correlation between the $\mathrm{Hb}$ values of the two methods. Figure 3 illustrates that there is a strong and highly positive correlation between the $\mathrm{Hb}$ results compared with the previous figures, and most of the $\mathrm{Hb}$ values are restricted between 12.0 and $16.0 \mathrm{~g} / \mathrm{dl}$.

\section{DISCUSSION}

An accurate measurement of $\mathrm{Hb}$ in the population is crucial to help evaluate the extent of anemia and to avoid wrongly rejecting or accepting blood donors in hospitals or blood bank centers. The main goal of assessing $\mathrm{Hb}$ is to ensure the prevention and supervision of anemia in any location. Several efforts have been done to guarantee that an appropriate technique comes out over the years that will contribute to the accuracy and quality of the standard laboratory methods and simultaneously provide a simple and fast result without any challenges. ${ }^{15,16}$ As a general rule, any volunteer individual who has a good health, a normal $\mathrm{Hb}$ level, and had no recent severe infection is suitable and could be accepted as a donor. Every transfusion service, however, follows a detailed policy that may be different slightly from place to place, but a few considerations such as an accurate machine for measuring $\mathrm{Hb}$ and well-trained staff should come first.

The HemoCue method is a widely used method for measuring $\mathrm{Hb}$. The HemoCue is a portable machine that directly measures the $\mathrm{Hb}$ from undiluted blood samples. In addition, the measurement of two-wavelengths by HemoCue has been found to be a new approach to correct the background turbidity of the samples and to give

Table 4. Bland and Altman approach for determining the agreement between pairs of the three methods used for Hb measurement

\begin{tabular}{lcccc}
\hline Reference method & Testing method & Mean of the difference & Limits of agreement & p-value for the difference \\
\hline Coulter LH-750 & HemoCue B-Hb & 1.0 & \pm 1.72 & $<0.001$ \\
Coulter LH-750 & HemoCue-301 & 0.7 & \pm 2.1 & $<0.001$ \\
HemoCue B-Hb & HemoCue-301 & 0.3 & \pm 1.5 & $<0.001$ \\
\hline
\end{tabular}




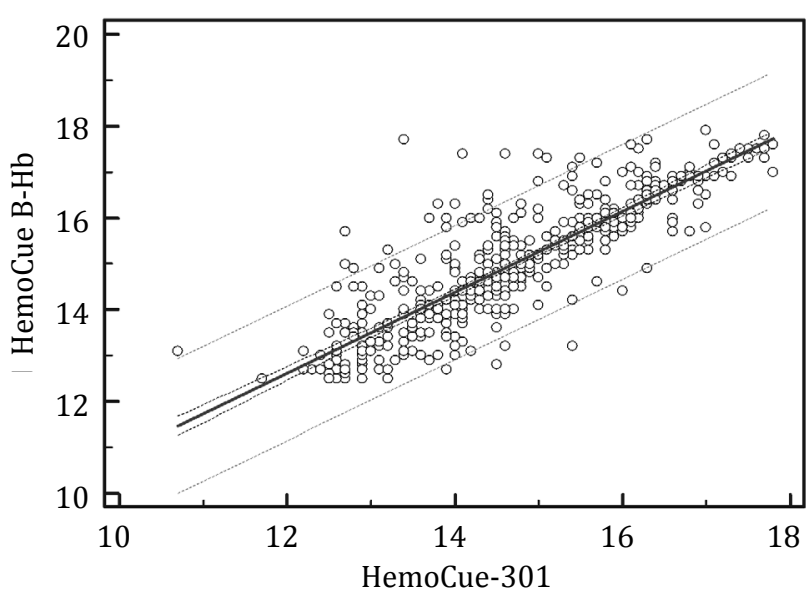

Figure 1. Scattergram of hemoglobin values $(\mathrm{g} / \mathrm{dl})$ in 455 donors, as determined by HemoCue B-Hb and HemoCue-301

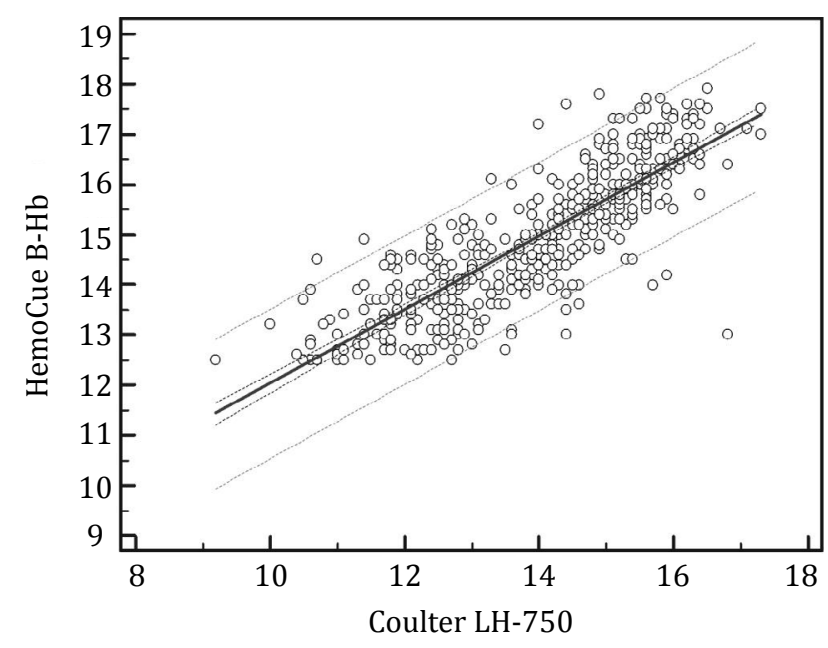

Figure 2. Scattergram of hemoglobin values (g/dl) in 455 donors, as determined by HemoCue B-Hb and Coulter LH750

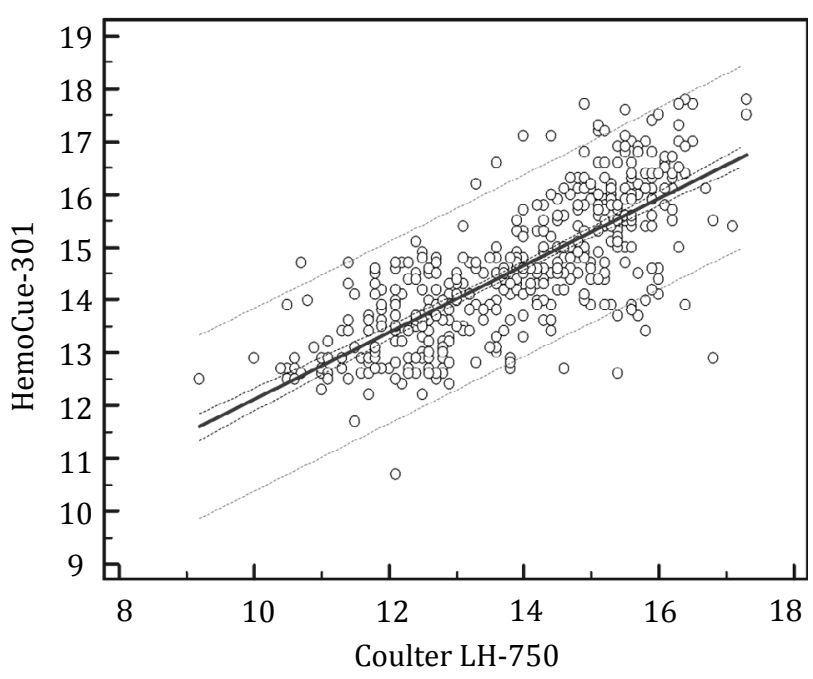

Figure 3. Scattergram of hemoglobin values (g/dl) in 455 donors, as determined by HemoCue-301 and Coulter LH-750 more accurate results. The present study shows that the $\mathrm{Hb}$ values obtained by the HemoCue methods (HemoCue B-Hb and HemoCue-301) were higher than the corresponding Coulter LH750 values, the differences being statistically significant. Our data indicate that both the HemoCue B-Hb and the HemoCue-301 methods show the best agreement when compared with the Coulter LH-750. However, pairs of methods involving the HemoCue $\mathrm{B}-\mathrm{Hb}$ and HemoCue-301 methods seem to have closer limits of agreement than the Coulter LH-750 and HemoCue combination, and statistically significant differences were detected between the $\mathrm{Hb}$ measurements of both methods $(\mathrm{p}<0.001)$.

A previous study in the UK that compared the $\mathrm{Hb}$ measurements of the HemoCue and Coulter STKS concluded that there was no statistically significant difference between the means by t-test. ${ }^{17}$ The mean $\mathrm{Hb}$ and the reference range of the HemoCue and Coulter methods in the present study were different from that of reported in the previous study by Rechner et al $^{11}$ (mean $=15.3$ and $15.2 \mathrm{~g} / \mathrm{dl}$ and the range=7.8-21.5 and 7.8-21.7 g/ dl for HemoCue and Coulter STKS, respectively). In addition, the means of the differences of the HemoCue B-Hb, HemoCue-301, and Coulter LH-750 in our study were $0.3,0.7$, and $1.0 \mathrm{~g} / \mathrm{dl}$, respectively. These are lower than the mean of differences mentioned in their study, which was $2.5 \mathrm{~g} / \mathrm{l}^{11}$, and higher than the $0.1 \mathrm{~g} / \mathrm{dl}$ reported by Rosenblit et al. ${ }^{17}$ An earlier study reported that a $\mathrm{Hb}$ difference greater than $1 \mathrm{~g} / \mathrm{dl}$ between the HemoCue and the Coulter STKS is considered clinically significant. ${ }^{16}$ The $1 \mathrm{~g} / \mathrm{dl}$ difference between the HemoCue system and the Coulter LH-750 can be explained by the fact that the HemoCue system compensates for turbidity in the blood sample. ${ }^{16} \mathrm{~A}$ similar study conducted in Brazil ${ }^{17}$ and another study ${ }^{18}$ reported that the HemoCue method and the Coulter method are statistically different, which is similar to our finding.

The different results found in various studies may be due to several factors, which can affect the degree of sensitivity and specificity and the level of agreement. These factors include the type of samples (venous or capillary), type of reagents, the concentration of anticoagulant, staff training, and weather and transportation conditions. In the current study, the same blood sample (venous) was used to assess the performance of the different $\mathrm{Hb}$ estimation 
methods, such as the HemoCue and Coulter LH-750. This helps us to evaluate the statistical differences and agreement that could occur in measuring the $\mathrm{Hb}$ level. Highly positive correlations were observed when we compared the two HemoCue methods against each other or against the Coulter LH-750, but it was stronger between the two HemoCue methods. Study samples were collected from Chinese high schools in the same area, which may be considered as not a representative sample because of the absence of rejected donors using the copper sulfate method or the HemoCue B-Hb method. Finally, the technique and the staff may have contributed to the differences, which occurred when measuring the $\mathrm{Hb}$ levels using the three methods. These factors may differ in various blood centers and may not be generalized to other communities.

In conclusion, this study shows that HemoCue B-Hb and HemoCue-301 analyzers, when properly and accurately used, will provide comparable accuracy of $\mathrm{Hb}$ estimation compared with the Coulter LH-750 method. However, the three methods reflect a good agreement for $\mathrm{Hb}$ determination. The differences between the results of this study and of the previous studies might be attributed to the variations in sample size, races, and locations as well as the type of blood sample used. Therefore, it is highly recommended to enroll a comprehensive population sample (Malay, Chinese, Indian, and others), use the same blood sample (venous), and include hospitals, medical centers, and schools in the representative sample to better reflect the accuracy and level of agreements between the three methods.

\section{Conflict of interest}

The authors affirm no conflict of interest in this study.

\section{Acknowledgment}

The authors would like to thank Advanced Medical and Dental Institute, Pusat Darah Negara, Universiti Sains Malaysia and the Ministry of Higher Education, Malaysia for providing financial support and research facilities.

\section{REFERENCES}

1. Gómez-Simón A, Navarro-Núñez L, Pérez-Ceballos E, Lozano, ML, Candela, MJ, Cascales, A, et al. Evaluation of four rapid methods for hemoglobin screening of whole blood donors in mobile collection settings. Transfus Apher Sci. 2007;36(3):235-42.

2. Tondon R, Verma A, Pandey P, Chaudhary R. Quality evaluation of four hemoglobin screening methods in a blood donor setting along with their comparative cost analysis in an Indian scenario. Asian J Transfus Sci. 2009;3(2):66-9.

3. Transfusion practice guidelines for clinical and laboratory personnel. 3rd Edition March, National Blood Centre, Ministry of Health. 2008.

4. International Nutritional Anaemia Consultative Group (INACG). Measurements of iron status: a report of the International Nutritional Anaemia Consultative Group. USA: INACG, 1985.

5. Newman B. Blood donor suitability and allogeneic whole blood donation. Transfus Med Rev. 2001;15(3):234-44.

6. Morris $S$, Ruel $M$, Cohen RJ, Dewey KG, de la Brière B, Hassan MN. Precision, accuracy, and reliability of hemoglobin assessment with use of capillary blood. Am J Clin Nutr. 1999;69(6):1243-8.

7. Neufeld L, García-Guerra A, Sánchez-Francia D, NewtonSánchez 0, Ramírez-Villalobos MD, Rivera-Dommarco J. Hemoglobin measured by Hemocue and a reference method in venous and capillary blood: a validation study. Salud Publica Mex. 2002;44(3):219-27.

8. Stone JE, Simmons WK, Jutsum PJ, Gurney JM. An evaluation of methods of screening for anaemia. Bull World Health Organ. 1984;62(1):115-20.

9. Hudson-Thomas M, Bingham KC, Simmons WK. An evaluation of the HemoCue for measuring haemoglobin in field studies in Jamaica. Bull World Health Organ. 1994;72(3):423-6.

10. von Schenck H, Falkensson M, Lundberg B. Evaluation of "HemoCue," a new device for determining hemoglobin. Clin Chem. 1986;32(3):526-9.

11. Rechner IJ, Twigg A, Davies AF, Imong S. Evaluation of the HemoCue compared with the Coulter STKS for measurement of neonatal haemoglobin. Arch Dis Child Fetal Neonatal Ed. 2002;86(3):F188-9.

12. Lin LI. A concordance correlation coefficient to evaluate reproducibility. Biometrics. 1989;45(1):255-68.

13. Lin LI. Assay validation using the concordance correlation coefficient. Biometrics. 1992;48(2):599-604.

14. Bland JM, Altman DG. Statistical methods for assessing agreement between two methods of clinical measurement. Lancet. 1986;327(8476):307-10.

15. Chen PP, Short TG, Leug DH, Oh TE. A clinical evaluation of the Haemocue haemoglobinometer using capillary, venous and arterial samples. Anaesth Intensive Care. 1992;20(4):497-500.

16. van den Broek NR, Ntonya C, Mhango E, White SA. Diagnosing anaemia in pregnancy in rural clinics: assessing the potential of the haemoglobin colour scale. Bull World Health Organ. 1999;77(1):15-21.

17. Rosenblit J, Abreu CR, Szterling LN, Kutner JM, Hamerschlak N, Frutuoso P, et al. Evaluation of three methods for haemoglobin measurement in a blood donor setting. Sao Paulo Med J. 1999;117(3):108-12.

18. Cable RG. Hb screening of blood donors: how close is close enough? Transfusion. 2003;43(3):306-8. 